\title{
SMOOTH STRUCTURES ON THE FIELD OF PREQUANTUM HILBERT SPACES
}

\author{
RÓBERT SZŐKE
}

\begin{abstract}
When there is a family of complex structures on the phase space, parametrized by a set $S$, the prequantum Hilbert spaces produced by geometric quantization, using the half-form correction, also depends on these parameters. This way we obtain a field of Hilbert spaces $p: H^{p r Q} \rightarrow S$. We show that this field can have natural inequivalent smooth Hilbert bundle structures.
\end{abstract}

\section{INTRODUCTION}

Let $M^{m}$ be an $m$-dimensional compact Riemannian manifold, that is the configuration space of a classical mechanical system. Geometric quantization intends to construct a Hilbert space (the quantum Hilbert space) associated to this system, in a natural way.

According to the recipe of Kostant and Souriau [Ko, [So, W], the first step in this process, is to pass to phase space $(N, \omega)$, where $\omega$ is a symplectic form, and then to choose a Hermitian line bundle with connection $E \rightarrow N$, with curvature $-i \omega$. The prequantum Hilbert space $H^{p r Q}$ then consists of the $L^{2}$ sections of $E$. For physical reasons this Hilbert space is too big and one needs to reduce its size. Hence the next step is to select a Hilbert subspace of $H^{p r Q}$. Frequently this is done with the help of a complex structure on $N$. If $\omega$ is a Kähler form, $E$ also inherits a holomorphic structure and one can take the quantum Hilbert space $H$ to be the holomorphic $L^{2}$ sections of $E$. Often one includes in this construction the so called half-form correction. Assume that the canonical bundle $K_{N}$ admits a square root $\kappa$. Then the corrected prequantum Hilbert space consists of the $L^{2}$ sections of $E \otimes \kappa$ and the corrected quantum Hilbert space $H^{\text {corr }}$ consists of the holomorphic $L^{2}$ sections of $E \otimes \kappa$.

In certain situations not only one but an entire family (parametrized by a set $S$ ) of complex structures exists on $N$ resulting a family of quantum Hilbert spaces $H_{s}$ (or $H_{s}^{\text {corr }}$ ). The question then arises: is it possible to identify these Hilbert spaces in a canonical way?

Suppose the set $S$ admits a smooth manifold structure. Axelrod et al. ADW and Hitchin Hi] suggest to view the family $\left\{H_{s}: s \in S\right\}$ (or $H_{s}^{c o r r}$ ) as the fibers of a Hilbert bundle $H \rightarrow S$ (resp. $H^{\text {corr }} \rightarrow S$ ) endowed with some Hermitian connection, the quantum connection. In situations when this is true, parallel transport along a curve in $S$ would yield a unitary map between different fibers. If furthermore the quantum connection was flat, we would even have a (locally) path-independent method of identification.

Now in the uncorrected situation $H^{p r Q}$ is fixed, it does not depend on the parameter $s$. Since $H_{s}$ are all closed subspaces of this Hilbert space, to implement the idea above, we could try to view this family as the fibers of a Hilbert subbundle

2000 Mathematics Subject Classification. 53D50, 53C35, 32L10, 70G45,65.

Key words and phrases. adapted complex structures, geometric quantization, Hilbert fields.

This research was supported by OTKA grant K112703. 
$H \rightarrow S$ of the trivial Hilbert bundle $\mathcal{L}:=S \times H^{p r Q} \rightarrow S$. In this case the quantum connection would arise from the canonical flat hermitian connection on $\mathcal{L}$ by orthogonal projection. In very specific situations ( $\mathrm{ADW}, \mathrm{W}]$ ) this works, but we are not aware of any general theorem that would guarantee that $H \rightarrow S$ is indeed a Hilbert subbundle of $L$.

In the corrected version a further difficulty arises: the prequantum Hilbert spaces $H_{s}^{p r Q}$ (the $L^{2}$ sections of $E \otimes \kappa_{s}$ ) themselves are no longer fixed but form a family of which the corrected quantum Hilbert spaces form a family of Hilbert subspaces.

Thus before we try to show that $\left\{H_{s}^{\text {corr }}, s \in S\right\}$ is a "subbundle" of $\left\{H_{s}^{p r Q}, s \in\right.$ $S\}$, we need to see whether $\left\{H_{s}^{p r Q}, s \in S\right\}$ forms a Hilbert bundle or not.

The main purpose of this note is to demonstrate that in certain cases there are at least two natural inequivalent ways to make $\left\{H_{s}^{p r Q}\right\}$ a Hilbert bundle. The topology on these bundles is the same, but their smooth structure (and therefore the set of smooth sections) are different. This is the content of Theorem 5.1.

Therefore it is better not to try to explain the smooth structure (and the quantum connection) on $H^{\text {corr }}$ (as in ADW, FMMN1, FMMN2 ) through $H^{p r Q}$ but rather do it directly, using the family of complex structures defined on $N$. This was our motivation and reason why we introduced in LSz14 the notion of smooth and analytic fields of Hilbert spaces generalizing Hilbert bundles with a hermitian connection. It is not clear whether $H^{\text {corr }} \rightarrow S$ really forms a smooth Hilbert subbundle or not in $H^{p r Q}$ equipped with either of the smooth structures considered in Theorem 5.1 .

\section{A unitary representation of $\mathcal{A}_{+}$}

The affine semigroup $\mathcal{A}$ is the semigroup of affine transformations $\sigma: \mathbb{R} \rightarrow \mathbb{R}$, $t \mapsto a+b t$, where $a, b \in \mathbb{R}$. Here $a$ and $b$ provide global coordinates on $\mathcal{A}$, and identify it with $\mathbb{R}^{2}$. With this single coordinate chart $\mathcal{A}$ becomes a Lie semigroup. The subset of $\mathcal{A}$ with $b>0$ forms a Lie (sub)group, that we shall denote by $\mathcal{A}_{+}$. In the above coordinates $\mathcal{A}_{+}$is identified, as a smooth manifold, with the upper half plane $S$.

$\mathcal{A}_{+}$acts naturally not only on $\mathbb{R}$ but on $\mathbb{C}$ as well. For an $s \in S$, let $\sigma_{s} \in \mathcal{A}_{+}$be the unique element with $\sigma_{s}(i)=s$. The map $S \rightarrow \mathcal{A}_{+}, s \mapsto \sigma_{s}$ is simply the inverse of the coordinate chart of $\mathcal{A}_{+}$.

The curves $\alpha, \beta: \mathbb{R} \rightarrow \mathcal{A}_{+}$defined as

$$
\alpha(u)=\{t \mapsto u+t\}, \quad \beta(u)=\left\{t \mapsto e^{u} t\right\}
$$

are 1-parameter subgroups in $\mathcal{A}_{+}$and $\dot{\alpha}(0), \dot{\beta}(0)$ form a basis in its Lie algebra. Define the homomorphism $\chi: \mathcal{A}_{+} \rightarrow(0, \infty)$ by $\chi(\sigma)=b$ if $\sigma(t)=a+b t$.

Let now $M^{m}$ be an $m$-dimensional, compact, smooth manifold equipped with a Riemannian metric. We take the phase space $N$ to be the manifold of parametrized geodesics $x: \mathbb{R} \rightarrow M$. Any $t_{0} \in \mathbb{R}$ induces a diffeomorphism $N \ni x \mapsto \dot{x}\left(t_{0}\right) \in T M$, and the pull back of the canonical symplectic form of $T M \approx T^{*} M$ is independent of $t_{0}$ (cf. [W] that we shall denote by $\omega$.

Associating with a point $q \in M$ the constant geodesic $\equiv q$, identifies $M$ as a submanifold in $N$. In the $T M$ picture this is just the zero section of the tangent bundle.

Composition of geodesics with the elements of $\mathcal{A}$ defines a right action of $\mathcal{A}$ on $N$. Induced by this action, we get the vector fields $\mathcal{X}, \mathcal{Y}$ on $N$, corresponding to $\dot{\alpha}(0)$ and $\dot{\beta}(0)$. When $N$ is replaced by $T M, \mathcal{X}$ is the vector field induced by the geodesic flow and $\mathcal{Y}$ corresponds to the Euler vector field. For $\sigma \in \mathcal{A}, x \in N$ let $A_{\sigma}: N \rightarrow N$ be defined by $A_{\sigma} x=x \circ \sigma$. As one easily checks,

$$
A_{\sigma}^{*} \omega=\chi(\sigma) \omega \text {. }
$$


Let $\mathcal{E}=\frac{\omega^{m}}{m !}$ be the Liouville volume form on $N$. From (2.2) we get

$$
A_{\sigma}^{*}(\mathcal{E})=\chi(\sigma)^{m} \mathcal{E} .
$$

Denote by $L^{2}(N, \mathcal{E})$ the complex valued square integrable (w.r.t. the volume form $\mathcal{E}$ ) functions on $N$ and $U\left(L^{2}(N, \mathcal{E})\right)$ the unitary self maps of this Hilbert space.

Theorem 2.1. With $\sigma \in \mathcal{A}_{+}$, the map

$$
\begin{array}{ccc}
\rho(\sigma): L^{2}(N, \mathcal{E}) & \rightarrow & L^{2}(N, \mathcal{E}) \\
f & \mapsto \chi(\sigma)^{\frac{m}{2}} f \circ A_{\sigma}
\end{array},
$$

is unitary and yields a unitary representation

$$
\rho: \mathcal{A}_{+} \rightarrow U\left(L^{2}(N, \mathcal{E})\right) .
$$

The map

$$
\varrho: \mathcal{A}_{+} \times L^{2}(N, \mathcal{E}) \longrightarrow L^{2}(N, \mathcal{E}),
$$

defined by $\varrho(\sigma, f):=\rho(\sigma) f$ is continuous, but not differentiable.

Proof. (2.3) implies

$$
\int_{N}|\rho(\sigma) f|^{2} \mathcal{E}=\int_{N} \chi(\sigma)^{m}\left|f \circ A_{\sigma}\right|^{2} \mathcal{E}=\int_{N} A_{\sigma}^{*}\left(|f|^{2} \mathcal{E}\right)=\int_{N}|f|^{2} \mathcal{E}
$$

hence $\rho(\sigma)$ in (2.4) is unitary. Also if $\sigma, \sigma^{\prime} \in \mathcal{A}_{+}$,

$$
\begin{gathered}
\rho\left(\sigma \sigma^{\prime}\right) f=\chi\left(\sigma \sigma^{\prime}\right)^{\frac{m}{2}} f \circ A_{\sigma \sigma^{\prime}}= \\
\chi\left(\sigma \sigma^{\prime}\right)^{\frac{m}{2}} f \circ\left(A_{\sigma^{\prime}} \circ A_{\sigma}\right)=\chi(\sigma)^{\frac{m}{2}}\left(\rho\left(\sigma^{\prime}\right) f\right) \circ A_{\sigma}=\rho(\sigma)\left(\rho\left(\sigma^{\prime}\right) f\right)
\end{gathered}
$$

shows that $\rho$ is a representation.

We prove the continuity of $\varrho$ in two steps.

First step: Let $g \in L^{2}(N, \mathcal{E})$ and $\sigma \in \mathcal{A}_{+}$be fixed. Assume that $g$ is continuous with compact support. We want to show that, when $f \in L^{2}(N, \mathcal{E})$ is close to $g$ and $\sigma^{\prime} \in \mathcal{A}_{+}$is close to $\sigma^{\prime}$, then $\rho\left(\sigma^{\prime}\right) f$ is close to $\rho(\sigma) g$. In the rest of the proof norm always refers to the $L^{2}$ norm w.r.t. the volume form $\mathcal{E}$.

$\left\|\rho\left(\sigma^{\prime}\right) f-\rho(\sigma) g\right\| \leq\left\|\rho\left(\sigma^{\prime}\right) f-\rho\left(\sigma^{\prime}\right) g\right\|+\left\|\rho\left(\sigma^{\prime}\right) g-\rho(\sigma) g\right\|=\|f-g\|+\left\|\rho\left(\sigma^{\prime}\right) g-\rho(\sigma) g\right\|$, since $\rho\left(\sigma^{\prime}\right)$ is unitary.

$$
\begin{aligned}
\left\|\rho\left(\sigma^{\prime}\right) g-\rho(\sigma) g\right\|^{2} & =\int_{N}\left|\chi\left(\sigma^{\prime}\right)^{\frac{m}{2}} g \circ A_{\sigma^{\prime}}-\chi(\sigma)^{\frac{m}{2}} g \circ A_{\sigma}\right|^{2} \mathcal{E}= \\
& =\int_{N} \chi(\sigma)^{m}\left|\left(\chi\left(\sigma^{\prime} \sigma^{-1}\right)\right)^{\frac{m}{2}} g \circ A_{\sigma^{-1} \sigma^{\prime}}-g\right|^{2} \circ A_{\sigma} \mathcal{E}= \\
& =\int_{N}\left|\left(\chi\left(\sigma^{\prime} \sigma^{-1}\right)\right)^{\frac{m}{2}} g \circ A_{\sigma^{-1} \sigma^{\prime}}-g\right|^{2} \mathcal{E},
\end{aligned}
$$

because $\rho(\sigma)$ is also unitary. This implies

$\left\|\rho\left(\sigma^{\prime}\right) g-\rho(\sigma) g\right\| \leq \sqrt{\int_{N}\left(\left(\chi\left(\sigma^{\prime} \sigma^{-1}\right)\right)^{\frac{m}{2}}-1\right)^{2}\left|g \circ A_{\sigma^{-1} \sigma^{\prime}}\right|^{2} \mathcal{E}}+\sqrt{\int_{N}\left|g \circ A_{\sigma^{-1} \sigma^{\prime}}-g\right|^{2} \mathcal{E}}$

Denote the two terms on the right hand side by $I$ and $I I$. Using the unitarity of $\rho\left(\sigma^{-1} \sigma^{\prime}\right)$ we get

$$
I=\chi\left(\sigma \sigma^{\prime-1}\right)^{\frac{m}{2}}\left|\chi\left(\sigma^{\prime} \sigma^{-1}\right)^{\frac{m}{2}}-1\right|\|g\|
$$

If $\sigma^{\prime}$ is close to $\sigma, \chi\left(\sigma^{\prime} \sigma^{-1}\right)$ is near to 1 and so $I$ is close to zero. Also in this case $A_{\sigma^{-1} \sigma^{\prime}}$ is close to the identity diffeomorphism of $N$. Because of our choice $g$ 
is uniformly continuous on its support. Consequently $I I$ is also near to zero. All these imply the continuity of $\Lambda$ in $(\sigma, g)$.

Second step: Let now $g \in L^{2}(N, \mathcal{E})$ be arbitrary. Choose a $g_{1}$ near $g$ that is continuous with compact support. Let $f \in L^{2}(N, \mathcal{E})$. Then

$$
\begin{gathered}
\left\|\rho\left(\sigma^{\prime}\right) f-\rho(\sigma) g\right\| \leq\left\|\rho\left(\sigma^{\prime}\right) f-\rho\left(\sigma^{\prime}\right) g\right\|+\left\|\rho\left(\sigma^{\prime}\right) g-\rho\left(\sigma^{\prime}\right) g_{1}\right\|+ \\
+\left\|\rho\left(\sigma^{\prime}\right) g_{1}-\rho(\sigma) g_{1}\right\|+\left\|\rho(\sigma) g_{1}-\rho(\sigma) g\right\| \\
=\|f-g\|+\left\|g-g_{1}\right\|+\left\|\rho\left(\sigma^{\prime}\right) g_{1}-\rho(\sigma) g_{1}\right\|+\left\|g_{1}-g\right\|
\end{gathered}
$$

and applying the first step to $g_{1}$ we get the continuity of $\varrho$ in $(\sigma, g)$.

To justify that $\varrho$ is not differentiable, it suffices to show that its partial derivative with respect to the $\sigma$ variable does not exist.

Consider the 1-parameter subgroups $\alpha, \beta$ in $\mathcal{A}_{+}$from (2.1) and let $g \in L^{2}(N, \mathcal{E})$ be arbitrary. Then $\varrho(\alpha(s), g)=g \circ A_{\alpha(s)}, \varrho(\beta(s), g)=e^{\frac{s m}{2}} g \circ A_{\beta(s)}$. So we would get

$$
\left.\frac{d}{d s}\right|_{s=0} \varrho(\alpha(s), g)=\mathcal{X} g,\left.\quad \frac{d}{d s}\right|_{s=0} \varrho(\beta(s), g)=\frac{m}{2} g+\mathcal{Y} g .
$$

Of course for a generic $g$ neither $\mathcal{X} g$, nor $\mathcal{Y} g$ exists and even if it does it is not necessarily square integrable.

\section{Adapted complex structures}

First we recall some important facts on adapted complex structures mainly from [LSz12]. If we are given a complex manifold structure on $\mathcal{A}_{+}$, a complex structure on $N$ is called adapted if for every $x \in N$ the orbit map $\mathcal{A}_{+} \ni \sigma \mapsto x \sigma \in N$ is holomorphic. An adapted complex structure on $N$ can exist only if the initial compex structure on $\mathcal{A}$ is left invariant. The left invariant complex structures on $\mathcal{A}$ are parametrized by the points of $\mathbb{C} \backslash \mathbb{R}$. For each $s \in \mathbb{C} \backslash \mathbb{R}$ and corresponding left invariant complex structure $I(s)$ on $\mathcal{A}$, if an $I(s)$ adapted complex structure $J(s)$ exists on $N$, then this structure is unique and if $J(i)$ exists, then $J(s)$ also exists for all $s$ in $s \in \mathbb{C} \backslash \mathbb{R}$. It is not easy to find such Riemannian manifolds $M$ for which $J(i)$ exists. The only known examples are: certain surfaces of revolution ([Sz91]), compact normal Riemannian homogeneous spaces Sz98, some examples obtained from the homogeneous examples using the method of symplectic reduction $(\widehat{\mathrm{Ag}})$. One can also take products or coverings of such spaces, but these are all we know so far.

The original definition of adapted complex structures in [LSz91] corresponds to the parameter $s=i$. Recall that $S$ denotes the upper half plane. For $s \in S$, $(N, \omega, J(s))$ becomes a Kähler manifold, denote its canonical bundle by $K^{s}$. If $s=i$, we simply write $K$ instead of $K^{i}$.

Proposition 3.1. Assume $M$ is oriented. Then the bundle $K^{s}$ is holomorphically trivial.

This is Lemma 10.4.1 in [LSz14], but for the readers' sake we include its short proof here.

Proof. According to [LSz12, Theorem 6c], the map $A_{\sigma_{s}}:(N, J(s)) \rightarrow(N, J(i))$ is a biholomorphism. Therefore it is enough to deal with the case $s=i$. Since $(N, J(i))$ is a Stein manifold ([LSz91, Theorem 5.6]), by the Oka principle $([\mathrm{H}, \mathrm{pp}$. 144-145], it suffices to show that $K$ is smoothly trivial. $M$ is a deformation retract in $N$, so we only need to show that $\left.K\right|_{M}$ is trivial. Since $M$ is oriented, the bundle $K_{M} \rightarrow M$ of real $m$-forms is trivial. But restricting a form in $\left.K\right|_{M}$ to $T M$ is an isomorphism $\left.K\right|_{M} \approx \mathbb{C} \otimes K_{M}$ and we are done. 
It is interesting, that although the canonical bundle $K$ is trivial, the first guess for a trivializing holomorphic section is wrong. Namely if one takes the $(\mathrm{m}, 0) \mathrm{com}-$ ponent of the volume form of $M$ and extend it holomorphically to $N$ (assuming that such an extension exists not only in a neighborhood of $M$ in $N$ ), this holomorphic $(\mathrm{m}, 0)$ form may have zeroes in $N \backslash M$, so it is not a trivializing section of $K$. This is the case for example for those surface of revolution metrics on the two sphere in Sz91.

The formula

$$
h_{s}(\alpha, \alpha) \mathcal{E}(x)=i^{m^{2}} \alpha \wedge \bar{\alpha} \quad x \in N, \alpha \in K_{x}^{s}
$$

defines a hermitian metric $h_{s}$ on $K^{s}$. Write $h$ for $h_{i}$.

Due to the proposition, when $M$ is orientable, there is a Hermitian holomorphic (in fact trivial) line bundle $\left(\kappa, h_{\kappa}\right)$ so that $\left(\kappa \otimes \kappa, h_{\kappa}^{2}\right) \approx(K, h)$. Let $\Theta$ be a trivializing holomorphic section of $K$ and $\theta$ the corresponding section of $\kappa$ with $\theta \otimes \theta=\Theta$. Taking $\kappa^{s}:=A_{\sigma_{s}}^{*} \kappa, h_{\kappa_{s}}=A_{\sigma_{s}}^{*} h_{\kappa}$, we have $\left(\kappa^{s} \otimes \kappa^{s}, h_{\kappa_{s}}^{2}\right) \approx\left(K^{s}, h_{s}\right)$. Let $\Theta_{s}:=A_{\sigma_{s}}^{*} \Theta$ and $\theta_{s}:=A_{\sigma_{s}}^{*} \theta$.

\section{Proposition 3.2.}

$$
h_{s}\left(\Theta_{s}, \Theta_{s}\right)=(\operatorname{Im} s)^{m} h(\Theta, \Theta) \circ A_{\sigma_{s}} \quad h_{\kappa_{s}}\left(\theta_{s}, \theta_{s}\right)^{2}=h_{s}\left(\Theta_{s}, \Theta_{s}\right)
$$

Proof. Since $\chi\left(\sigma_{s}\right)=\operatorname{Im} s,(3.1)$ and (2.3) implies

$\Theta_{s} \wedge \bar{\Theta}_{s}=A_{\sigma_{s}}^{*}(\Theta \wedge \bar{\Theta})=A_{\sigma_{s}}^{*}\left(h(\Theta, \Theta)(-i)^{m^{2}} \mathcal{E}\right)=(-i)^{m^{2}}(\operatorname{Im} s)^{m}\left(h(\Theta, \Theta) \circ A_{\sigma_{s}}\right) \mathcal{E}$

Using (3.1) once again we obtain the first formula from which the second follows from the definitions.

\section{The Field of PREQUANTUm Hilbert SPACES}

The prequantum line bundle is a Hermitian line bundle $E \rightarrow N$ with a hermitian connection whose curvature is $-i \omega$. When $M$ is simply connected, it is unique. In any case one such line bundle is the trivial line bundle $E=N \times \mathbb{C} \rightarrow N$ with the trivial metric $h^{E}(x, \gamma)=|\gamma|^{2}$ on it. The connection on $E$ is obtained from a real 1 -form $a$ on $N$ such that $d a=-\omega$ (such $a$ exists since $\omega$ is the pull back of the canonical symplectic form of $T^{*} M$ ). Let $\vartheta$ be the canonical section of $E$ i.e. that is defined by $\vartheta(x):=(x, 1), x \in N$. Then all the sections of $E$ have the form $f \vartheta$, where $f: N \rightarrow \mathbb{C}$ and the connection on $E$ is defined by

$$
\nabla_{\zeta}^{E}(f \vartheta)=(\zeta f+i a(\zeta)) \vartheta
$$

where $\zeta$ is any smooth complex vector field on $N$. The half-form corrected prequantum Hilbert space $H_{s}^{p r Q}$ corresponding to the Kähler manifold $(N, \omega, J(s))$, $s \in S$ is the Hilbert space of $L^{2}$ sections of the bundle $E \otimes \kappa^{s}$. Since $\vartheta \otimes \theta_{s}$ is a nowhere vanishing section of $E \otimes \kappa^{s}$,

$$
H_{s}^{p r Q}=\left\{\psi=\left.f_{s} \vartheta \otimes \theta_{s}\left|f_{s}: N \rightarrow \mathbb{C}, \int_{N}\right| f_{s}\right|^{2} h_{\kappa_{s}}\left(\theta_{s}, \theta_{s}\right) \mathcal{E}<\infty\right\} .
$$

Let

$$
H^{p r Q}:=\cup_{s \in S}^{*} H_{s}^{p r Q}
$$

be the disjoint union of these Hilbert spaces. $p: H^{p r Q} \rightarrow S$ is the natural projection map. At the moment there is no further structure defined yet on the set $H^{\operatorname{pr} Q}$, except that the fibers $p^{-1}(s)$ are Hilbert spaces. $p: H^{p r Q} \rightarrow S$ is an example that we call (cf. [LSz14]) a field of Hilbert spaces. 


\section{Smooth Hilbert Bundle Structures on $H^{p r Q}$}

The map

$$
\begin{array}{clc}
\mathcal{L}:=S \times L^{2}(N, \mathcal{E}) & \stackrel{A}{\longrightarrow} & H^{p r Q} \\
(s, f) & \longmapsto & \frac{f}{\sqrt{h_{\kappa_{s}}\left(\theta_{s}, \theta_{s}\right)}} \vartheta \otimes \theta_{s}
\end{array}
$$

is a fiber preserving bijection and its restriction to each fiber is unitary. Therefore pushing forward $\mathcal{L}$ with $A$, equips $H^{p r Q}$ with a smooth (in fact) trivial Hilbert bundle structure. The canonical flat hermitian connection on $\mathcal{L}$ yields an orthogonal connection on $H^{p r Q}$.

When $M$ is a compact Lie group equipped with a biinvariant metric, this is the Hilbert bundle structure with hermitian connection chosen by C. Florentino, P. Matias, J. Mourão and J. Nunes in their papers [FMMN1, FMMN2, except that they do not consider the full parameter space $S$, only the positive imaginary axes.

But this is not the only possible natural way to equip $H^{\text {prQ }}$ with a Hilbert bundle structure. Let $\psi_{s}=f_{s} \vartheta \otimes \theta_{s} \in H_{s}^{p r Q}$. Then from Proposition 3.2 we get

$$
\begin{gathered}
\left\|\psi_{s}\right\|_{L^{2}}^{2}=\int_{N}\left|f_{s}\right|^{2} h_{\kappa_{s}}\left(\theta_{s}, \theta_{s}\right) \mathcal{E}=(\operatorname{Im} s)^{\frac{m}{2}} \int_{N}\left|f_{s}\right|^{2}\left(\sqrt{h(\Theta, \Theta)} \circ A_{\sigma_{s}}\right) \mathcal{E}= \\
=(\operatorname{Im} s)^{-\frac{m}{2}} \int_{N} A_{\sigma_{s}}^{*}\left(\left|f_{s}\right|^{2} \circ A_{\sigma_{s}}^{-1} \sqrt{h(\Theta, \Theta)} \mathcal{E}\right)=(\operatorname{Im} s)^{-\frac{m}{2}} \int_{N}\left(\left|f_{s}\right|^{2} \circ A_{\sigma_{s}}^{-1}\right) \sqrt{h(\Theta, \Theta)} \mathcal{E}
\end{gathered}
$$

Therefore the map

$$
\begin{array}{ccc}
H^{p r Q} & \stackrel{B}{\longrightarrow} & \mathcal{L}=S \times L^{2}(N, \mathcal{E}) \\
f_{s} \vartheta \otimes \theta_{s} & \longmapsto & \left(s,(\operatorname{Im} s)^{-\frac{m}{4}} f_{s} \circ A_{\sigma_{s}}^{-1} h(\Theta, \Theta)^{\frac{1}{4}}\right)
\end{array}
$$

is a fiber preserving bijection whose restriction to each fiber is unitary. By pulling back the Hilbert bundle structure of $\mathcal{L}$ with $B$, the Hilbert field $p: H^{p r Q} \rightarrow S$ inherits another smooth (in fact trivial) Hilbert bundle structure. We claim that as a smooth bundle, this is different from the one we obtained with the help of the map $A$ earlier.

Theorem 5.1. The Hilbert bundle structures on $p: H^{p r Q} \rightarrow S$ obtained by the maps $A$ and $B$ are the same as topological Hilbert bundles but their smooth structures are different.

Proof. Using Proposition 3.2 we calculate the map $B \circ A: \mathcal{L} \rightarrow \mathcal{L}$ to be

$$
(s, f) \mapsto\left(s,(\mathrm{Im} \mathrm{s})^{-\frac{m}{2}} f \circ A_{\sigma_{s}}^{-1}\right)=\left(s, \varrho\left(\left(\sigma_{s}\right)^{-1}\right) f\right) .
$$

It follows from Theorem 2.1 that the fiberwise unitary map $B \circ A$ is a homeomorphism but it is not differentiable.

Acknowledgement We thank László Lempert for stimulating discussions.

\section{REFERENCES}

[ADW] S. Axelrod, S. Della Pietra, E. Witten: Geometric quantization of Chern-Simons gauge theory, J. Diff. Geo., 33, 1991, 787-902

[Ag] R.M. Aguilar: Symplectic reduction and the homogeneous complex Monge-Ampére equation, Ann. Glob. Anal. Geom. 19, 2001, 327-353

[FMMN1] C. Florentino, P. Matias, J. Mourão, J.P. Nunes: Geometric quantization, complex structures and the coherent state transform, J. Funct. Anal., 221, 2005, 303-322

[FMMN2] C. Florentino, P. Matias, J. Mourão, J.P. Nunes: On the BKS pairing for Kähler quantizations of the cotangent bundle of a Lie group, J. Funct. Anal., 234, 2006, 180198

[Hi] N. Hitchin: Flat connections and geometric quantization, Comm. Math. Phys., 131, 1990, 347-380 
[H] L. Hörmander: An introduction to complex analysis in several complex variables, 3rd ed., North Holland, Amsterdam, 1991

[Ko] B. Kostant: Quantization and unitary representations I., Lectures in modern analysis and applications III, Lecture Notes in Math., 170, Springer, Berlin, 1970, 87-208

[LSz91] L. Lempert, R. Szöke: Global solutions of the homogeneous complex Monge-Ampère equation and complex structures on the tangent bundle of Riemannian manifolds, Math. Ann., 290, 1991, 689-712

[LSz12] L. Lempert, R. Szőke: A new look at adapted complex structures, Bull. Lond. Math. Soc., 44, 2012, 367-374

[LSz14] L. Lempert, R. Szőke: Direct images, fields of Hilbert spaces, and geometric quantization, Comm. Math. Phys., 327, 2014, 49-99

[So] J.-M. Souriau: Structure des systèmes dynamiques, Dunod, Paris, 1970

[Sz91] R. Szőke: Complex structures on tangent bundles of Riemannian manifolds, Math. Ann., 291, 1991, 409-428

[Sz98] R. Szőke: Adapted complex structures and Riemannian homogeneous spaces, Ann. Polon. Math., LXX, 1998, 215-220

[W] N.M.J. Woodhouse: Geometric quantization, 2nd ed., Clarendon Press, Oxford, 1992

Department of Analysis, Institute of Mathematics, ELTE EÖtvös Loránd University, PÁzmány PÉter sétány 1/C, Budapest 1117, Hungary, ORCiD:0000-0002-8723-1068

E-mail address: rszoke@cs.elte.hu 